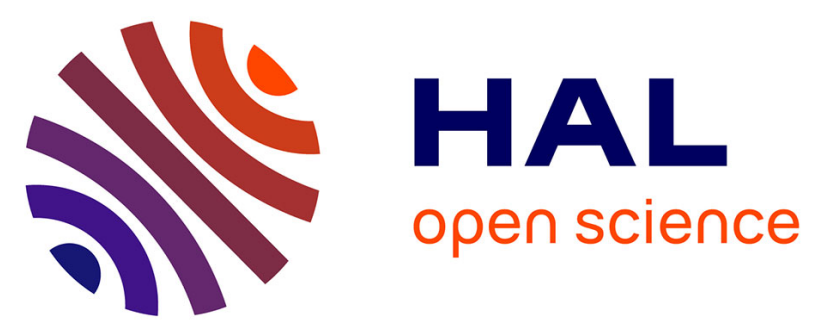

\title{
GAMA : a simulation platform that integrates geographical information data, agent-based modeling and multi-scale control
}

Patrick Taillandier, Duc-An Vo, Edouard Amouroux, Alexis Drogoul

\section{- To cite this version:}

Patrick Taillandier, Duc-An Vo, Edouard Amouroux, Alexis Drogoul. GAMA : a simulation platform that integrates geographical information data, agent-based modeling and multi-scale control. PRINCIPLES AND PRACTICE OF MULTI-AGENT SYSTEMS, 2012, Kolkata, India. pp.242-258, 10.1007/978-3-642-25920-3_17. hal-00688318v2

\section{HAL Id: hal-00688318 \\ https://hal.science/hal-00688318v2}

Submitted on 19 Apr 2012

HAL is a multi-disciplinary open access archive for the deposit and dissemination of scientific research documents, whether they are published or not. The documents may come from teaching and research institutions in France or abroad, or from public or private research centers.
L'archive ouverte pluridisciplinaire HAL, est destinée au dépôt et à la diffusion de documents scientifiques de niveau recherche, publiés ou non, émanant des établissements d'enseignement et de recherche français ou étrangers, des laboratoires publics ou privés.

\section{(c)(1)}

Distributed under a Creative Commons Attribution| 4.0 International License 


\title{
GAMA: a simulation platform that integrates geographical information data, agent-based modeling and multi-scale control
}

\author{
Patrick Taillandier ${ }^{1,2}$, Duc-An $\mathrm{Vo}^{1,2}$, Edouard Amouroux ${ }^{1,2}$, \\ and Alexis Drogoul ${ }^{1,2}$, \\ ${ }^{1}$ IRD, UMI UMMISCO 209, \\ 32 avenue Henri Varagnat, 93143 Bondy, France \\ ${ }^{2}$ IFI, MSI, UMI 209, \\ ngo 42 Ta Quang Buu, Ha Noi, Viet Nam \\ patrick.taillandier@gmail.com, voducanvn@yahoo.com, edouard.amouroux@ird.fr, \\ alexis.drogoul@gmail.com
}

\begin{abstract}
The agent-based modeling is now widely used to study complex systems. Its ability to represent several levels of interaction along a detailed (complex) environment representation favored such a development. However, in many models, these capabilities are not fully used. Indeed, only simple, usually discrete, environment representation and one level of interaction (rarely two or three) are considered in most of the agent-based models. The major reason behind this fact is the lack of simulation platforms assisting the work of modelers in these domains. To tackle this problem, we developed a new simulation platform, GAMA. This platform allows modelers to define spatially explicit and multi-levels models. In particular, it integrates powerful tools coming from Geographic Information Systems (GIS) and Data Mining easing the modeling and analysis efforts. In this paper, we present how this platform addresses these issues and how such tools are available right out of the box to modelers.
\end{abstract}

Keywords: Simulation platform, Agent-based modeling, Geographical vector data, Multi-level control

\section{Introduction}

The agent-based modeling has brought a new way to study the complex systems. It allows to take into account different levels of interactions as well as the heterogeneity of the entities composing the system.

Even if numerous simulation platforms exist, most of the complex models are still developed from scratch. Indeed, very few platforms allow to directly work with geographical vector data (series of coordinates defining geometries) and/or to define multi-level models. Moreover, these platforms are often complex to use and their understanding can require a time investment from the modeler that can be similar to the one needed to develop a model from scratch. 
In this paper, we present the GAMA agent-based simulation platform [1], [2]. This platform provides a complete modeling and simulation development environment for building spatially explicit multi-agent simulations. Many models have already been implemented using this platform (e.g. [3][4][5][6]). Its main advantages come from its versatility (domain independent) and the simplicity to define a model with it. Indeed, GAMA provides a rich, yet accessible, modeling language based on XML, GAML, that allows to define complex models integrating at the same time entities of different scales and geographical vector data.

The paper is organized as follow. In Section 2, we present the capabilities of GAMA concerning the integration of geographical vector data. Section 3 is dedicated to the presentation of its multi-scale modeling capabilities. In Section 4, we investigate a way to couple the use of geographical data and multi-scale modeling. Section 5 discusses about the contributions of this paper. At last, Section 6 concludes.

\section{Integrating Geographical Vector Data in Simulation}

\subsection{Why using geographical vector data in models?}

These last years have seen the development on a large scale of geographical vector datasets. Today, most of the decision makers use this type of data when they have to face a problem integrating a spatial dimension.

In the context of simulations, using this type of data allows to make the simulations closer to the field situation. In addition, it allows to use tools, like spatial analysis, coming from Geographic Information Systems (GIS) to manage these data.

\subsection{Use of geographical data in models}

If more and more models integrate geographical vector data, their use can take different forms. In the following sections, we present three different ways of using vector data, from the simplest (reading/writing of geographical data) to the most complex (agentification of geographical data).

\subsubsection{Reading and writing of geographical vector data}

The most basic functions concerning the use of geographical vector data are the reading and the writing of geographical data from files and from database. The goal is to integrate seamlessly the vector data as the simulation's environment (input) and to store the resulting environment (output).

Once geographical vector data has been read, several uses can be made of them. The most straightforward one consists in translating them as a grid where agents are localized.

\subsubsection{Using geographical vector data as background layers}

A more complex use consists in using these data as a "background layer" constituted of geographical objects: the agents will be able to move according to this layer. For 
example, some agents will be able to move along a network of road, or inside a complex polygon (e.g. inside a forest represented by a polygon.

This use requires the integration in the simulator of GIS specific primitives such as moving an agent inside a geometry, computing a shortest path between two points of this geometry (or on a network), etc.

\subsubsection{Agentification of Geographical vector data}

A richer ways of integrating geographical vector data in a model is to consider each geographical object as an agent. Thus, a road will be an agent, a building or a city, and each object contained in a geographical dataset will also be represented by an agent. Remark that this kind of geographical data agentification was already used for other application contexts such as cartographic generalization [7]. In the context of simulation, the advantage of this approach is to give the possibility to manage geographical objects exactly like other agents in the simulation: it will be possible to give them an internal state and a behavior.

Reciprocally, it is possible to go further and to consider that every "spatialized" (localized and with a geometry) agents of the simulation has a geometry and can be viewed as a geographical object in a geographical dataset. In this way, the management of agents and geographical objects is equivalent and trouble-free. Indeed, no difference is made anymore between agents and geographical objects.

\subsection{Geographical vector data in existing simulation platforms}

\subsubsection{Simulation platforms with basic support of geographical vector data}

Swarm [8] is a well-established simulation platform and inspiration for many others. Its original version does not allow to integrate geographical vector data. However, a library called Kenge [9] allows to load layers of geographical vector data. Practically, this extension allows to create a cellular automata from a shape file. In addition, an ad hoc access to geographical data has been developed for specific models (e.g. [10]). Unfortunately, they do not provide any spatial primitives neither the possibility to store the resulted environment.

Netlogo [11] is also a well-established simulation platform. It is largely used for educational purpose and for research. The GIS support has been added recently through an extension [12]. It allows import and export of vector data and support the projection system (the method used to represent the geographical data on a plane). The attributes of the vector data are made accessible as well as their geometrical characteristics (centroid, list of vertex, etc.). Some basic geometrical operations are also available (bounding rectangles, union of polygons, etc.). However, many more advanced spatial analysis operation are not offered.

CORMAS [13] is a platform dedicated to the modeling in ecology and especially the natural resources management where space representation and interaction is essential. It proposes two environment modes: vector and raster. They share the same organization of 3 classes «spatial entity», «agent», and «object». This organization, though being rigid, ease the development of model by abstracting the interaction with environment, thus allows to switch from a discrete environment to a continuous (or 
vector) one. Unfortunately, CORMAS provides only basic services for the discrete environment. Moreover, GIS support is limited to loading and storing shapefiles (a popular vector data format) and creating elementary areas. GIS primitives (union, intersection, shortest path, etc.) and access to polygon attributes have to be programmed.

In 2008, Urbani proposed the SMAG (portmanteau word from SMA-SIG or MASGIS in English) architecture linking a GIS and MABS simulator for decision support system. The author implemented it over CORMAS, calling it CORMGIS [14]. The integration is relatively basic as access to geo-referenced data is done through a dataconnection to ArcGIS. In addition, no GIS primitive (union, intersection, etc) is available.

\subsubsection{Simulation platforms with advanced support of geographical vector data} Repast $\mathbf{J}$ [15] is a modeling toolkit inspired by Swarm. As a toolkit, it provides a structure with only basic services readily available. Different grids are implemented (hexagonal or rectangular, torus or not, etc.) but agents are not (only an interface is given). The GIS support is done through the OpenMap library. It provides the minimal services of a GIS: importing/exporting shapefiles and raster data, some geometrical operations, access to data attributes, etc. Nevertheless, as Repast J provides access to OpenMap, the modeler can implement more complex operations. Unfortunately, this programming is far from reach of the vast majority of modelers.

Repast Symphony (Repast S) [16] is the up-to-date version of the Repast toolkit. It provides the same basic features as Repast $\mathbf{J}$, but is based on a more advanced GIS library, Geotools, which provides additional GIS services. In particular, Repast S allows to directly model a network of lines as a graph and to compute the shortest paths from one point to another. It allows as well to visualize and manage 3D data. Nevertheless, the number of GIS operations available is still fairly limited and localized agents are still to be programmed. More advanced operations have to be programmed (using the Geotools librabry) which is again, evidently, far from reach for many modelers.

\subsection{Geographical vector data in GAMA}

In order to address these shortcomings we developed the GAMA platform, which goes much further by making available many more GIS services and operations and especially an advance management of geographical vector data.

The first version of GAMA that was presented in [1] proposed the idea of using a continuous environment to serve as a reference for all other environments (e.g. grid environment). In this former version, all situated agents had a point for geometry. The use of geographical vector data was very limited: there were just to initialize the initial location of the agents and as a background layer.

If the new version of GAMA (GAMA 1.3) kept the same idea of a reference environment, it goes further by providing a true geometry to all situated agents. This geometry, which is based on vector representation, can be simple (point, polyline or polygon) or complex (composed of several sub-geometries). 
The geometry of the agents can be defined by the modeler (a list of points) or directly loaded from a shapefile. Indeed, GAMA allows to use geographical vector data to create agents of a specific species (a prototype of agents that defines both the agent internal state and their behavior): each object of the geographical data will be automatically used to instantiate an agent, GAMA taking care of managing the spatial projection of the data and, if necessary, of reading the values of the attributes. Consequently GAMA considers localized agents and geographical objects in the exact same way.

Example: the following GAML lines allow to create a set of building agents from the shapefile shape_file_building.shp and to set the value of the attribute nature of each created building agent according to the attribute NATURE of the shapefile:

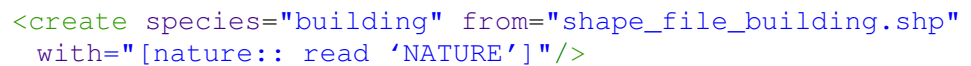

Figure 1 gives an example of the agentification of 4 buildings from a shapefile.

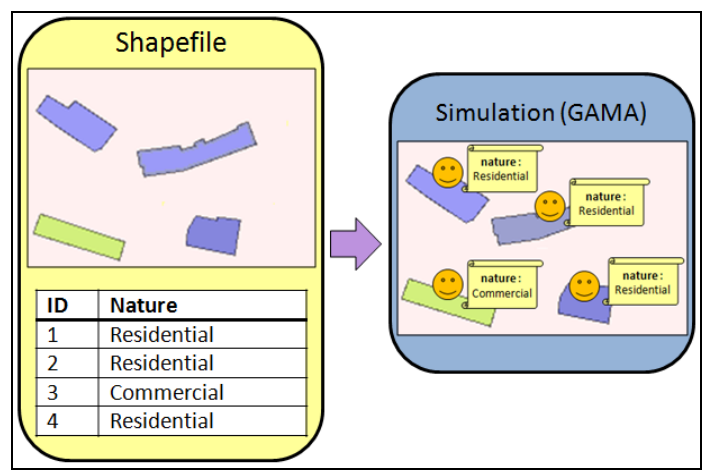

Fig. 1. Example of geographical data agentification

In the same way, GAMA allows to save a set of agents in a shapefile.

Example: the following GAML lines allow to save all the agents of the species building in the shapefile shape_file_building.shp and to set the value of the attribute NATURE of each geographical object according to the attribute nature of the agents:

$$
\text { <save species="building" to="shape_file_building.shp" }
$$

with=" [nature: : 'NATURE'] "/>

In order to ease the manipulation of the vector geometries, GAMA integrates different GIS features that are directly available through the GAML language. Thus, GAMA allows to:

- Compute the area and the perimeter of a geometry.

Example: The following GAML line allows to compute the area of the geometry of the agent $a g$ :

<let name="the_area" value="ag.area" />

- Test if two geometries intersect, touch, cross, overlap each other.

Example: The following GAML lines allow to test if the geometry of the agent that is applying the action intersects the geometry geom:

\footnotetext{
$<$ do action="interection" return="is_true" >$$
</ \text { do }\rangle
$$

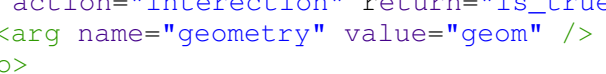


- Compute the convex hull and the buffer geometry of a geometry (Figure 2).

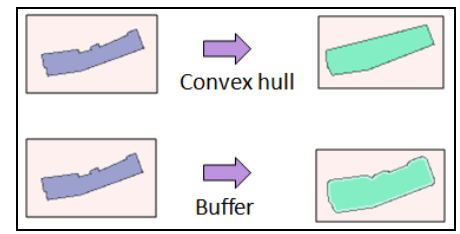

Fig. 2. Example of convex hull and buffer actions.

Example: The following GAML line allows to compute the convex hull of the geometry of the agent that is applying the action:

<do action="convex_hull" return="result" / >

- Apply translation, rotation and scaling operations on a geometry (Figure 3).

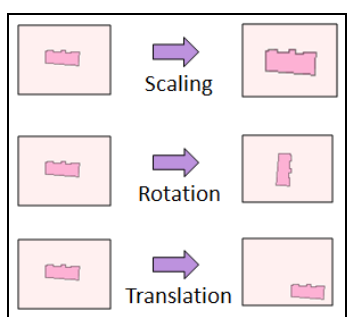

Fig. 3. Example of scaling, rotation and translation actions.

Example: The following GAML lines allow to rotate the geometry of the agent that is applying the action with an angle of $90^{\circ}$ :

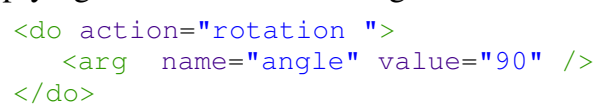

- Compute the geometry resulting from the union, intersection or difference of two geometries (Figure 4).

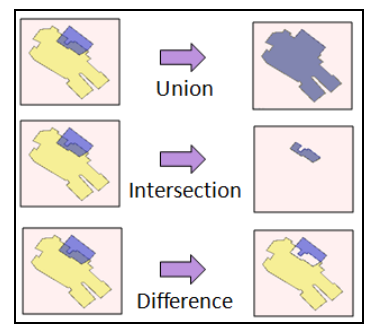

Fig. 4. Example of union, intersection and difference actions.

Example: The following GAML lines allow to compute the difference between the geometry geom $_{1}$ and the geometry geom 2 :

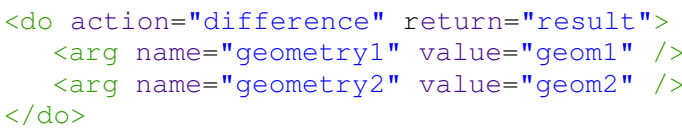


- Compute the distance between two geometries (minimal distance).

Example: The following GAML lines allow to compute the distance between the geometry of the agent that is applying the action and the geometry geom:

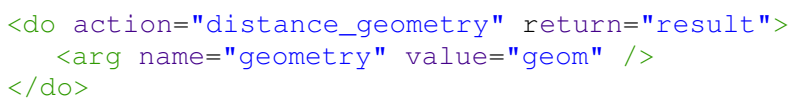

- Compute the neighborhood of an agent, i.e. all the agents that are localized at a distance lower than a given thresholds to the agent.

Example: The following GAML lines allow to compute the neighborhood of the agent $a g$ :

<let name="neighborhood" value="ag.neighbours_geometry "/>

- Compute a random point inside a geometry.

Example: The following GAML lines allow to compute a random point inside the geometry geom:

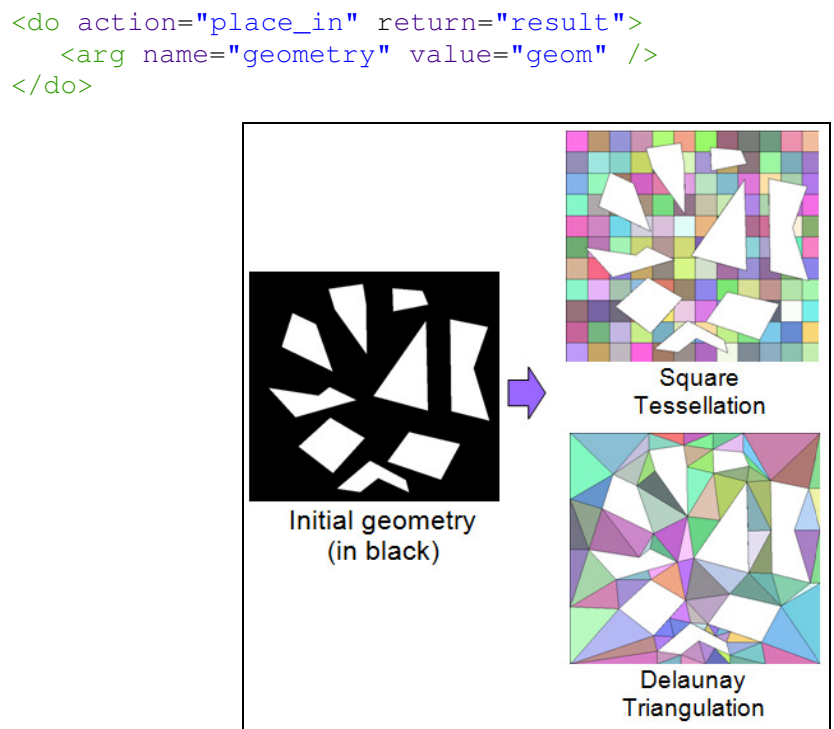

Fig. 5. Example of Tessellations (square and triangle).

- Compute the point of a geometry that is the closest to the agent location.

Example: The following GAML lines allow to compute the point of the geometry geom that is the closest to the agent that is applying the action.

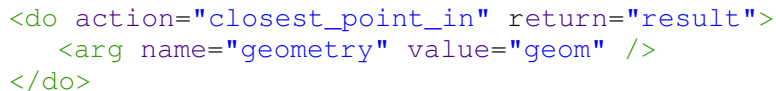

- Apply a tessellation operation (square or triangle) on a geometry (Figure 5).

Example: The following GAML lines allow to compute the Delaunay triangulation of the geometry (polygon) geom:

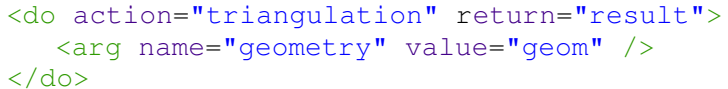

- Compute the skeleton of a geometry (Figure 6). 


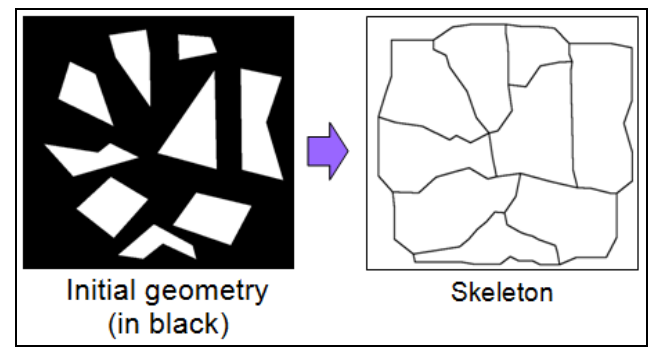

Fig. 6. Example of Skeletonization.

Example: The following GAML lines allow to compute the skeleton of the geometry (polygon) geom:

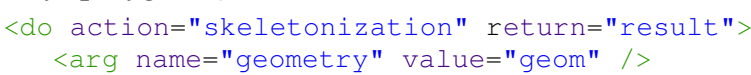

- Compute the shortest path (or the distance) inside a geometry (line network or polygon) between two points located in the geometry. For this computation, our approach consists in modeling the geometry as a graph, and in computing from it the shortest path linking the two points. In the context of a line network, the modeling as a graph is trivial. In the context of a polygon, this one is based on a Delaunay triangulation of the geometry: each triangle resulting from the triangulation is modeled as a node and an edge represents the fact that two triangles are adjacent. Figure 7 shows an example of graph computation. Two algorithms are implemented for the shortest path computation: Dijkstra [17] and Floyd Warshall [18].

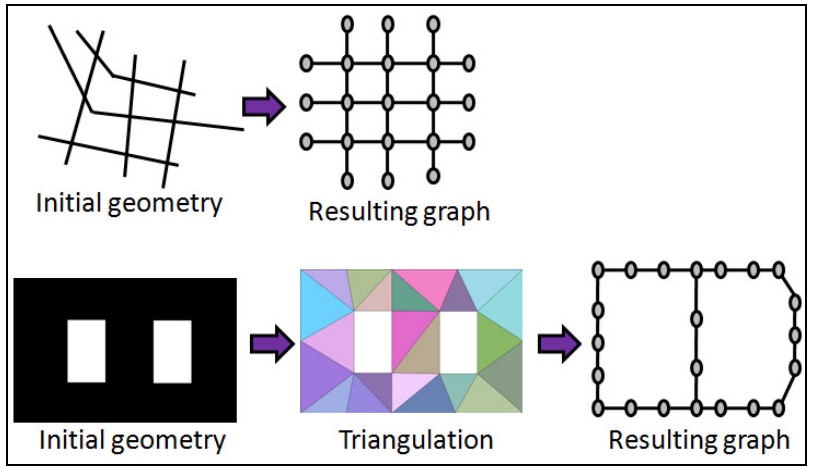

Fig. 7. Example of graph computation

Example: the following GAML lines allow to move the agent that is applying the action toward the point the_target, at a speed of $5 \mathrm{~km} / \mathrm{h}$, inside the geometry geom (which can be a graph or a polygon):

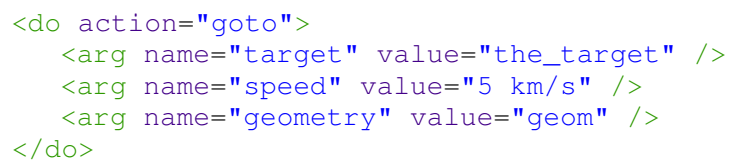




\section{Multi-scale Modeling}

\subsection{Context}

Another advantage of the agent-based modeling approach is its representation versatility. Indeed, an "agent" can represent any individual or aggregation/structure of individuals of the reference system, at any spatial scale and across different time horizons. Thus the modeler is free in her/his choice of the entities of the reference system that will be represented by agents. This choice will depend on the level of abstraction of the reference system the modeler is working with. This, in turn, depends on the question he/she wants to answer with the model, on the data available at hand, on the scale at which this data is described, etc.

In addition to the agent representing entities of the reference system, the modeler can need to explicitly represent emergent structures. Indeed, during the simulation stage (execution of the model), some structure can emerge: appearance of pheromone trail built by ant [19], evolution of social group within a population [20], formation of arches in granular environment [21], etc. These structures are often the result of nonlinear interactions between the agents defined in the model and can play a significant role in the model dynamics. They can be considered as a higher level of abstraction (upper scale) compare to the underlying agents composing them. It is important, if not crucial, to be able to detect and to generate them dynamically (i.e. might simplified the simulation run).

Current agent-based modeling platforms lack support in term of agent-based modeling language to represent these structures as explicit entities in the model and tools to detect them. Thus, modelers face difficulties when they need to represent them and to follow their dynamics during the course of the simulation.

\subsection{Multi-scale modeling in GAMA}

In GAMA, in order to let modelers dynamically track the emergence of dynamic structures, we let them represent these structures as explicit entities in the model. We call these entities "emergent agents". As regular agent, an emergent agent can have attributes and behaviors. Beside, its instantiation depends on the appearance of certain properties during the simulation and its life-cycle possesses some specific operations.

\subsubsection{Representing emergent structure}

The "creation" operation helps to specify when an emergent agent is instantiated. This operation allows the modeler to express in an explicit way the rules governing the instantiation of emergent agents during the simulation. For example, consider a simulation of city dynamics: a modeler can decide to instantiate an emergent agent of species building block when two or more building agents are close enough. Figure 8 illustrates this example: an emergent agent (building block) representing the emergent structure is created with six micro-agents (building) as components. 


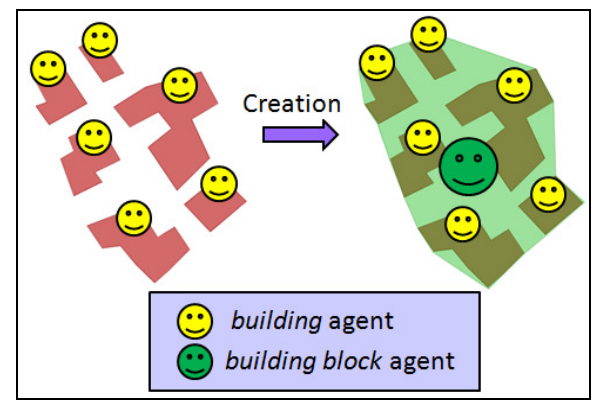

Fig. 8. Creation of an emergent agent (building block agent)

The "update" operation describes how micro-agents are added to or removed from an emergent agent. Some micro-agents may no longer satisfy a condition to belong to an emergent agent, while others, still "free" may now fulfill it: this operation allows to specify how these agents are added or removed from the structure. The purpose of this operation is to keep the list of components up-to-date with respect to the meaning of the emergent agent.

Figure 9 illustrates the "update" operation. It follows the example of city dynamic simulation presented Figure 8. We consider that a building block agent composes of three building agents. One building agent doesn't satisfy the condition to belong to the building block agent anymore. A free building agent satisfies the condition to become a member of the building block agent. This operation helps the modeler to remove one building agent from the building block agent and add one building agent to the building block agent.

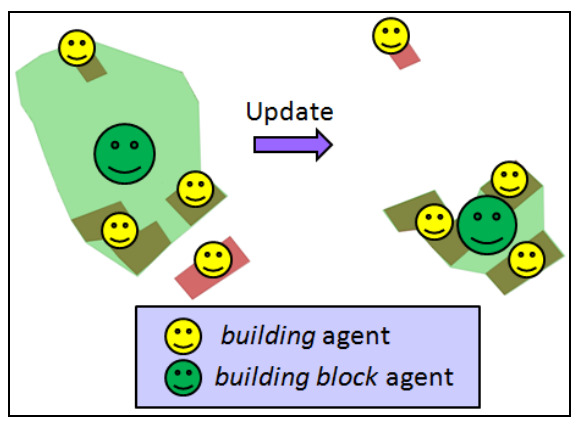

Fig. 9. Update of an emergent agent (building block agent)

The "merge" operation allows the modeler to specify how several emergent agents representing different structures can be merged into one unique emergent agent. The fusion of their respective components then becomes the components of the new unique emergent agent.

Figure 10 illustrates the "merge" operation using the same example as Figure 8 and 9. We consider a new building block agent (in yellow) has been created. This agent is close enough to the existing building block agent (in green) to merge with it. The resulting agent will be composed of the 5 building agents composing the two building block agents. 


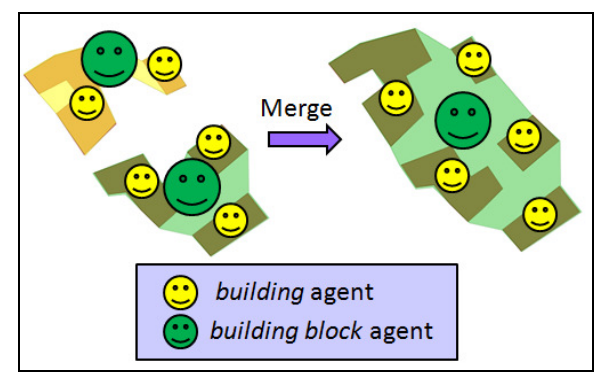

Fig. 10. Fusion of different emergent agents

The purpose of the "disposal" operation is to express when an emerging structure should not consider to be an agent in the simulation anymore. The emergent agent representing the structure is cleared out of the simulation and its components become free.

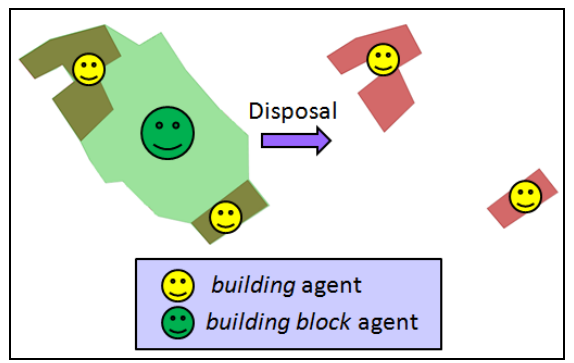

Fig. 11. Death of an emergent agent

Figure 11 illustrates the "disposal" operation. Following the example presented Figure 10, we consider that three of the building agents composing the building block agent died. Now, the remaining building agents are too far from each other to compose a building block agent. Then, the building block agent is going to die.

The top-down feedback control allows the modeler to describe which feedback constraint an emergent agent is exercising on its underlying micro-agents. As emergent agents usually emerge because of the interactions of certain micro-agents, these agents have an influence on its attributes and behavior. Reciprocally, an emergent agent may also provide a feedback on the behavior of its components, either implicitly or explicitly. In order to describe it, the modeler needs to have some way to alter the behavior of a micro-agent (by changing parameters, adding, or removing entire behaviors) before and after it enters an emergent agent.

Typically, in our city dynamic simulation example, a building agent, once part of building block agent, has more chance to attract residents to live in, and thus to lead to construction of new buildings in the neighborhood (for example, shops).

\subsubsection{Representing emergent agents in GAMA}

An emergent agent is composed of constituent agents. Constituent agents can be considered as micro-agents compared to the emergent agent. Reciprocally, the 
emergent agent can be seen as a macro-agent compared to its constituent agents. In turn, several emergent agents can be merged to form another emergent agent at a higher level of abstraction. Thus, an agent in GAMA can play the role of macro-agent in one level of organization and micro-agent in a higher level of abstraction. This design aims at permitting the modeler to represent as many levels of abstraction as he needs in his model. Figure 12 shows an example of abstraction level hierarchy for the city dynamic simulation problem: a city agent is composed of a set of district agents that are each composed of a set of building block agents that are at their turn composed of a set of building agents.

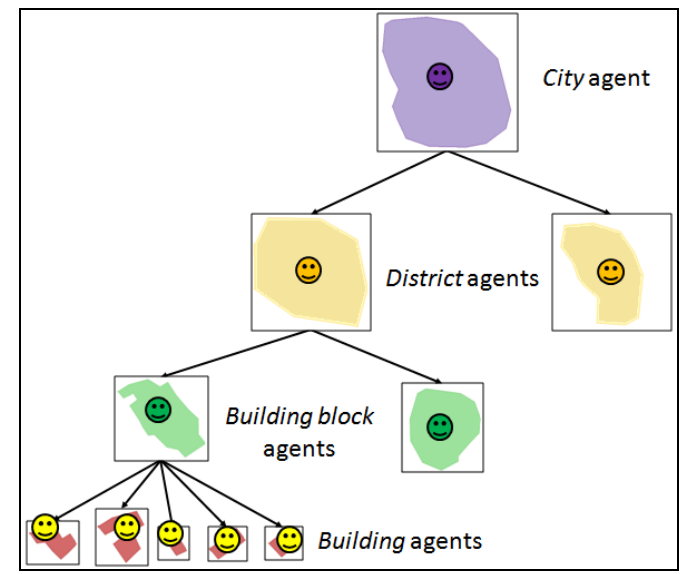

Fig. 12. Example of level of abstraction hierarchy

To manipulate the five specific operations in the lifecycle of an emergent agent (create, update, merge, disposal, top-down constraint control), six GAML commands are defined: creation, update, merge, disposal, enable and disable.

- The creation command allows to specify when emergent agents are created in the simulation.

Example: the following GAML lines create a building block agent which has for components the building agent contained in the list list_buildings:

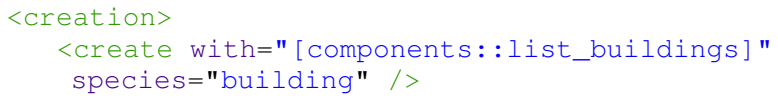

- The update command allows the modeler to define how the constituent microagents are added and removed from an emergent agent.

Example: the following GAML lines update the components of the building block agent that is applying this command by adding the building agents contained in added_buildings and removing the ones contained in removed_buildings:

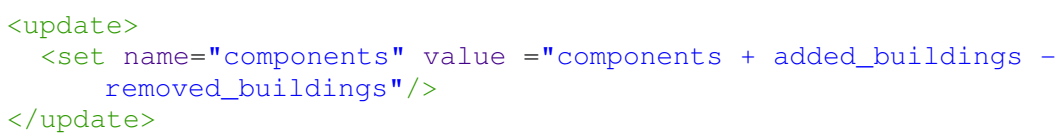

- The merge command allows the modeler to define how several emergent agents are merged. 
Example: the following GAML lines allow to merge several building block agents (the ones contained in the nearby_bb list) with the building block agent applying this command. All the constituent building agents of the building block agents contained in the nearby_bb list are added to the component list of the one applying the command. Then, the other building block agents die (i.e. are removed from the simulation):

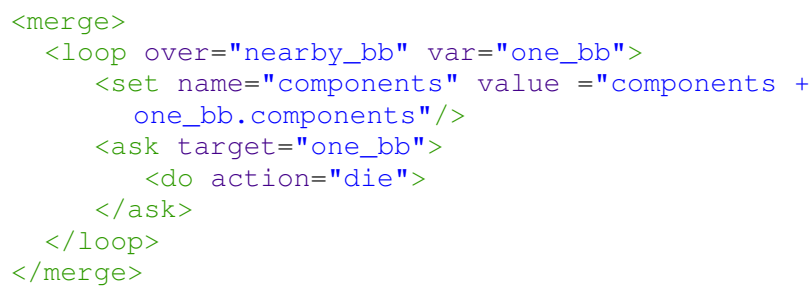

- The disposal command allows the modeler to specify when an emergent agent is cleared out of the simulation.

Example: the following GAML line specifies that a building block agent will be removed from the simulation if it contains less than two building agents:

$<$ isposal when=" (length components) $<2 " />$

- The disable command allows the modeler to disable certain behavior units appropriately. While the enable command allows the modeler to enable the inactive behavior units.

Example: the following GAML lines enable the behavior "expansion" and disable the behavior "destruction" of the building agent one_building_agent:

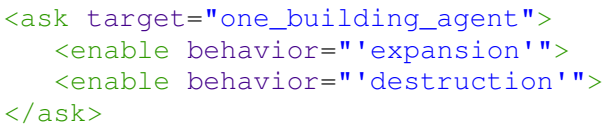

Note that GAMA provides several clustering algorithms (e.g. hierarchical clustering, X-Means [22], Cobweb [23]) that can be used to dynamically detect if an emergent agent has to be instantiate. For example, these algorithms can be used to detect groups of close agents, or agents sharing some specific attributes.

Example: the following GAML lines allows to regroup the building agents contained in the buildings list into a set of groups; each group being composed of building agents of which the distance to each other is lower or equal to $10 \mathrm{~m}$ :

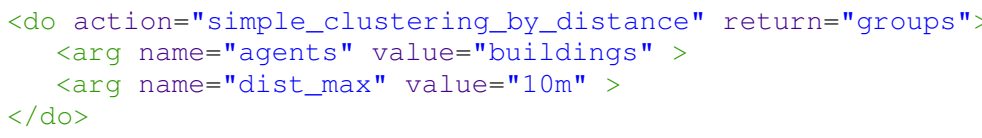

\section{Coupling Geographical Vector Data and Multi-scale modeling}

In Section 2.4, we presented the GIS capacities and in Section 3.2 its multi-scale modeling capacities of GAMA. In this section, we investigate a way to couple the use of geographical data and multi-scale modeling: we propose to decompose an agent into a set of constituent agents on geometric basis. One of the main interests of such 
decomposition is to improve the dynamicity of the special operations applied on the agent.

Indeed, consider an agent with a geometry, which is used to constraint the movement of other agents: for example, a road network agent on which some people agents are moving, a forest agent in which animal agents are moving, etc.. Moving agents on this geometry requires to compute a new graph from the geometry each time it is modified. This computation can be very time consuming if the geometry is complex. An approach to face this problem is to decompose the agent in a set of constituent agents on a geometric basis: each constituent agent will represent a part of the macro agent geometry (for example, a line in the context of line network, or a triangle in the context of a polygon). Instead of computing the new graph each time the geometry is modified, the complete graph will be computed only once and each constituent agent will remember its role in the graph. Then, each time the macro agent geometry is modified, it will locally update its list of micro agents (delete the micro agents which geometry is no more part of the global geometry, create new ones if necessary and modify the geometry of existing ones), and each micro agent will update its role in the graph. Figure 13 gives an example, where a graph was already computed for a geometry, and where the modification of the geometry has lead to a local update of the graph.

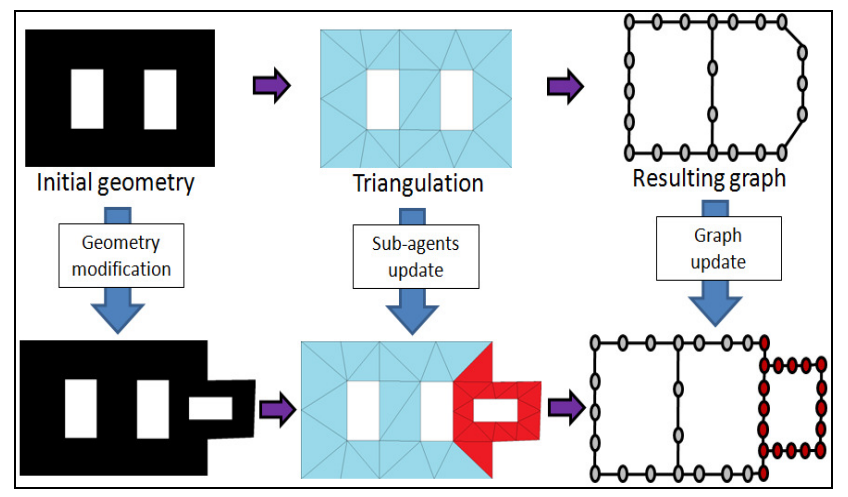

Fig. 13. Example of a local modification of a graph

In GAMA, using such an approach can be easily achieved. Indeed, in Section 2.4, we presented how GAMA allows to compute the square or triangle tessellation of a geometry and a graph from a geometry. More-over, as seen in Section 3.2, GAMA allows to define macro-agents (emergent agent). Thus, GAMA provides all the features that are required to apply this approach.

\section{Discussion}

We see the contributions of this work as threefold:

1. There is a difference between an idea and its implementation. What we incorporate into GAMA are implementations of ideas that may have been (or not) already 
proposed by other people but rarely found their way into operational instances. They are implemented into the platform and linked with the modeling language, so that they can be used by anyone building a model in GAMA. In our point of view, these implementations are contributions to the field, because they eliminate the ambiguities and the lack of formalism often found in ABM/MAS contributions and, most important, can be experimented.

2. Integrating existing techniques in a framework and enabling the researchers to easily choose the most appropriate is a delicate exercise. In GAMA, we have ensured that all the proposed techniques are tightly coupled, and that they are usable even by novice users through GAML. This allows us to build, in the same platform, simple models (a la NetLogo) alongside more complex models. Actually, our efforts of integration tend to the point that there are no real differences between a "simple" and a "complex" model. So, while it is true that, for instance, we did not invent graph-related techniques, we believe we contribute to the field by providing a way, for researchers, to use the most appropriate, transparently, into their models.

3. Following the previous point, we see GAMA as a contribution by itself, filling the gap between NetLogo, interesting for prototyping small models, but which does not scale well when it comes to real ones, and RePast, more a complete toolbox than a platform. The fact, for instance, that every agent in GAMA is provided with a geometry, and that any environment can be discretized, means that researchers can begin with a simple prototype (where agents are points on a grid, like in Netlogo) to test the logic of a model, and turn this model into a more realistic one, for example by loading data from a GIS base, without having to change anything to the logic. This radically transforms the experimental processes of ABM.

\section{Conclusion}

In this paper, we present the new advance features included in the last version of the GAMA platform (version 1.3)[2]. These features concern the use of geographical vector data and the definition of multi-scale models.

This version of GAMA is already used in several projects related to different application domains such as the avian flu local propagation in North Vietnam, the rift valley fever in Senegal, the brown hopper invasion in South Vietnam, the effect of emotions on waves of panic.

The next version of GAMA, version 1.4, is going to include a new integrated development environment (IDE) with a new modeling language. The goal is to ease the work of the modelers by providing a less extensive and easier to learn language. This version will also include all the classic features provide by most of the modern IDE (auto-completion, automatic detection of errors, etc.). In addition, we plan to improve the integration of the approach proposed in Section 4. Practically, for the moment, the use of this approach with GAMA is still complex and require much GAML code. Methods allowing to automate this approach are required. 


\section{References}

1. Amouroux, E., Chu, T. Q., Boucher, A., Drogoul, A.: GAMA: An Environment for Implementing and Running Spatially Explicit Multi-agent Simulations. PRIMA07, 359-371 (2007)

2. GAMA platform, http://gama-platform.googlecode.com

3. Amouroux, E., Desvaux, S., Drogoul, A.: Towards Virtual Epidemiology: An Agent-Based Approach to the Modeling of H5N1 Propagation and Persistence in North-Vietnam. PRIMA2008: 26-33 (2008)

4. Nguyen Vu, Q. A., Gaudou, B., Canal, R., Hassas, S.: Coherence and robustness in a disturbed MAS. IEEE-RIVF, DaNang, VietNam,. IEEE (2009)

5. Chu, T. Q., Drogoul, A., Boucher, A., Zucker J.: Interactive Learning of Independent Experts' Criteria for Rescue Simulations. Journal of Universal Computer Science, vol. 15, no. 13, 2701-2725 (2009)

6. Taillandier, P., Buard, E.: Designing Agent Behaviour in Agent-Based Simulation through participatory method. PRIMA, Nagoya, Japan, 571-578 (2009)

7. Ruas, A., Duchêne, C.: A prototype generalisation system based on the multi-agent system paradigm. Generalisation of Geographic information: cartographic modelling and applications, Elsevier Ltd, 269-284 (2007)

8. Minar, N., Burkhart, R., Langton, C., Askenazi M.: The Swarm Simulation System: A Toolkit for Building Multi-Agent Simulations, SFI Working Paper 96-06-042 (1996)

9. Box, P.: Spatial Units as Agents. Integrating GIS and Agent-Based Modelling Techniques. ed. Oxford (2002)

10.Haklay, M., O'Sullivan, D., Thurstain-Goodwin, M., Schelhorn, T.: So Go Downtown": Simulating Pedestrian Movement in Town Centres, Environment and Planning B: Planning and Design, 28(3): 343-359 (2001)

11.Wilensky, U.: NetLogo. http://ccl.northwestern.edu/netlogo/. Center for Connected Learning and Computer-Based Modeling, Northwestern University. Evanston, IL (1999)

12.Russell, E., Wilensky, U.: Consuming spatial data in NetLogo using the GIS Extension. The annual meeting of the Swarm Development Group. Chicago, IL (2008)

13.Bousquet, F., Bakam, I., Proton, H., Le Page, C. : Cormas: common-pool resources and multi-agents systems. IEA/AIE (Vol. 2) 826-837 (1998)

14.Urbani, D., Delhom, M.: Analyzing Knowledge Exchanges in Hybrid MAS GIS Decision Support Systems, Toward a New DSS Architecture, LNCS 4953, 323-332 (2008)

15.North, M. J., Collier, N. T., Vos, J. R.: Experiences Creating Three Implementations of the Repast Agent Modeling Toolkit. ACM Transactions on Modeling and Computer Simulation, Vol. 16, Issue 1, 1-25 (2006)

16.North, M. J., Tatara, E., Collier, N. T., Ozik, J.: Visual Agent-based Model Development with Repast Simphony, Conference on Complex Interaction and Social Emergence (2007)

17.Dijkstra, E. W.: A short introduction to the art of programming. Technological Univ. Eindhoven, Rep. EWD316 (1971)

18.Floyd, R.W. Algorithm 97: Shortest Path, Communications of the ACM, vol. 5, no 6, p. 345 (1962)

19.Camazine, S. et al. Self-Organization in Biological Systems. Princeton University Press, Princeton (2001)

20.Schelling, http://web.mit.edu/www/lab/alife/schelling.html

21.Laurent Breton et al.: A Multi-Agent Based Simulation of Sand Pile in Static Equilibrium. $M A B S(2000)$

22.Pelleg, D., Moore, A. W.: X-means: Extending K-means with Efficient Estimation of the Number of Clusters. International Conference on Machine Learning, 727-734 (2000)

23.Gennari, J. H., Langley, P., Fisher, D.: Models of incremental concept formation. Artificial Intelligence. 40:11-61 (1990) 